\title{
Qualification for endoscopic retrograde cholangiopancreatography in the diagnosis and treatment of extrahepatic cholestasis caused by choledocholithiasis
}

\author{
Krzysztof Kujawski ${ }^{1,2}$, Magdalena Stasiak ${ }^{3}$, Jacek Rysz ${ }^{2}$
}

\author{
${ }^{1}$ Gastrointestinal Endoscopy Laboratory, WAM University Hospital, Medical University \\ of Lodz, Lodz, Poland \\ 2Department of Nephrology, Hypertension and Family Medicine, Medical University \\ of Lodz, Lodz, Poland \\ ${ }^{3}$ Department of Endocrinology and Metabolic Diseases, Polish Mother's Memorial \\ Hospital-Research Institute, Lodz, Poland
}

Submitted: 14 August 2014

Accepted: 25 December 2014

Arch Med Sci 2015; 11, 6: 1213-1216

DOI: 10.5114/aoms.2015.56347

Copyright (C 2015 Termedia \& Banach

\section{Abstract}

Introduction: Choledocholithiasis, being the most common cause of extrahepatic cholestasis, is diagnosed on the basis of clinical symptoms, laboratory findings, and imaging results. An important diagnostic and also therapeutic procedure performed in patients with choledocholithiasis is retrograde cholangiopancreatography (ERCP). However, due to the high rate of complications associated with ERCP, the decision on its implementation should be preceded by a thorough analysis of the case, aimed at confirmation of the diagnosis. Material and methods: The present study is a retrospective analysis of 86 patients qualified for ERCP due to suspected choledocholithiasis. The diagnosis was based on clinical symptoms and/or laboratory and/or imaging results. The presence of one or more of the three abovementioned criteria was a key to classify patients in one of three risk groups of choledocholithiasis: high, intermediate and low.

Results: In the high-risk group, where choledocholithiasis was confirmed by clinical symptoms, laboratory findings and results of imaging tests, the accuracy of diagnosis was $100 \%$. In the intermediate-risk group - choledocholithiasis diagnosed on the basis of clinical symptoms and laboratory results - the accuracy of diagnosis was approximately $81.5 \%(p<0.05)$. In the lowrisk group, in which choledocholithiasis was diagnosed only on the basis of clinical symptoms, diagnosis accuracy was approximately $50 \%(p<0.05)$.

Conclusions: The combination of clinical symptoms, laboratory tests and imaging findings allows patients to be classified into three risk groups of choledocholithiasis: high, intermediate and low. Taking into account the probability of choledocholithiasis and the risk of ERCP complications, only patients from high and intermediate risk groups should be qualified for the procedure.

Key words: endoscopic retrograde cholangiopancreatography, choledocholithiasis, extrahepatic cholestasis.

\section{Introduction}

Extrahepatic cholestasis (synonym: obstructive jaundice) is a condition characterized by blocking of the bile outflow from the bile duct into

\author{
Corresponding author: \\ Krzysztof Kujawski \\ Gastrointestinal Endoscopy \\ Laboratory \\ WAM University Hospital \\ Medical University of Lodz \\ 113 Żeromskiego St \\ 90-549 Lodz, Poland \\ Phone: +48 (42) 6393568 \\ Fax: +48 (22) 6393782 \\ E-mail: kkujawski@poczta.fm
}


the duodenum. The consequence of difficult passage of bile is the presence of a number of clinical symptoms such as jaundice (yellowish pigmentation of the skin, sclerae and mucous membranes), dark urine, discolored stools, itching, abdominal pain or elevated body temperature. The clinical picture depends on the exact cause of cholestasis and duration of the disease [1, 2]. In order to determine the initial cause of the symptoms of cholestasis, laboratory tests and imaging should be performed. Blood tests that may help to identi-

Table I. Incidence of complications after ERCP (\%) [16-20]

\begin{tabular}{|lcc|}
\hline Complications & $\begin{array}{c}\text { Patients at } \\
\text { average risk of } \\
\text { the procedure }\end{array}$ & $\begin{array}{c}\text { Patients at } \\
\text { high risk of } \\
\text { the procedure }\end{array}$ \\
\hline Pancreatitis & 3 & 8 \\
\hline Bleeding & 0.2 & 0.4 \\
\hline Perforation & 0.1 & 0.3 \\
\hline Infection & 0.1 & 2 \\
\hline $\begin{array}{l}\text { Cardiorespiratory } \\
\text { complications }\end{array}$ & 0.5 & 2 \\
\hline Total & 3.9 & 12.7 \\
\hline
\end{tabular}

Table II. Indications for therapeutic ERCP

Biliary tract diseases:

- Jaundice or cholestasis with suspected obstructive nature

- Acute cholangitis

- Lesions in biliary tracts visualized by other imaging methods

- Biliary fistula or leakage

- latrogenic bile duct injury

Pancreatic diseases:

- Recurrent acute pancreatitis of uncertain etiology

- Severe acute biliary pancreatitis

- Pancreatic cancer - to collect tissue samples and apply palliative treatment

- Pancreatic fistula or leakage

- Pancreatic insufficiency, malabsorption

- Chronic pancreatitis with pain, jaundice or leakage

- Pancreatic pseudocyst

- Abdominal pain of pancreatic origin confirmed by laboratory tests or imaging

Endoscopic therapy:

- Endoscopic sphincterotomy/papillotomy

- Drainage of biliary/pancreatic tract

Endoscopic tissue and fluid collection:

- Brush biopsy, fine needle aspiration biopsy

- Collection of bile/pancreatic juice

Preoperative duct mapping:

- Malignant tumors

- Mild stenosis

- Chronic pancreatitis

Manometry:

- Sphincter of Oddi

- Duct stenosis fy the type of jaundice include the levels of alanine aminotransferase (ALT), aspartate aminotransferase (AST), alkaline phosphatase (ALP), total bilirubin and bilirubin fractions as well as $\gamma$-glutamyl transpeptidase (GGT).

If clinical signs and laboratory results indicate extrahepatic cholestasis, imaging should be performed, starting with simple abdominal ultrasound (US) examination. The presence of bile duct dilatation confirms extrahepatic cholestasis. In every case of the lack of bile duct dilatation in US, further, more precise imaging studies such as computed tomography (CT), magnetic resonance imaging (MRI) or endoscopic ultrasound (EUS) are required to prove the presence of stones in the bile ducts.

Endoscopic retrograde cholangiopancreatography (ERCP) is a method of biliary and pancreatic tract imaging. The image of contrasted biliary tracts obtained during ERCP is often sufficient to establish the diagnosis and introduce the treatment [3-6]. Sensitivity of ERCP in diagnostics of choledocholithiasis ranges from $79 \%$ to $95 \%$, with specificity reaching $92-98 \%[7,8]$. The procedure, however, is associated with a significant risk of complications (Table I) [9-11].

Due to a high rate of complications, this procedure should be introduced only if it is highly probable that it would be associated with a therapeutic effect [12-14]. Indications for therapeutic ERCP are shown in Table II $[15,16]$.

In every patient, a decision about ERCP should be preceded by a thorough analysis of the case in terms of the benefits and risks of complications.

\section{Material and methods}

The present study is a retrospective analysis of the criteria for qualification for ERCP in 86 patients who underwent the procedure for suspected choledocholithiasis. Three categories of indications were evaluated: clinical condition, results of laboratory tests and imaging studies. To assess the clinical status of the patient, the symptoms of obstructive cholestasis were analyzed, including yellowing of the skin and sclerae, itching, color of urine and stool. Additionally, clinical parameters assessing the respiratory and circulatory function were evaluated to ensure safety of ERCP performance. Among the laboratory tests the following parameters were taken into account: AST, ALT, total bilirubin and its fractions, GGT, ALP and tumor markers including Ca 19-9 and alpha-fetoprotein. The imaging studies analyzed in qualification for ERCP included abdominal US, as well as - in some cases - abdominal CT or MRI.

\section{Statistical analysis}

Statistical analysis was performed with the use of Statistica program. 
Table III. Relationship between the eventual presence of choledocholithiasis confirmed by ERCP and the clinical symptoms and/or laboratory and/or imaging results

\begin{tabular}{|lccc|}
\hline Indications & No. of performed ercp & $\begin{array}{c}\text { No. of confirmed cases } \\
\text { of choledocholithiasis }\end{array}$ & $\begin{array}{c}\text { Percent of correct } \\
\text { qualifications }\end{array}$ \\
\hline $\begin{array}{l}\text { - Clinical symptoms + } \\
- \text { Laboratory results + } \\
- \text { Imaging + }\end{array}$ & 51 & 51 & 100 \\
\hline $\begin{array}{l}\text { - Clinical symptoms + } \\
- \text { Laboratory results + } \\
- \text { Imaging - }\end{array}$ & 27 & 22 & 81.5 \\
\hline $\begin{array}{l}\text { - Clinical symptoms + } \\
- \text { Laboratory results - } \\
- \text { Imaging - }\end{array}$ & 8 & 4 & 50 \\
\hline
\end{tabular}

\section{Results}

In all 51 patients in whom clinical symptoms as well as both laboratory and imaging abnormalities were present before ERCP, the procedure appeared to be fully justified. In the group of 27 patients with clinical symptoms and laboratory abnormalities but without any pathological findings in imaging studies, in 5 cases ERCP was only a diagnostic procedure and did not confirm the presence of deposits in the bile ducts.

In the group of patients who were qualified for ERCP only on the basis of clinical status (without any confirmation of cholestasis in laboratory or imaging results) in 4 out of 8 patients, ERCP confirmed the presence of deposits in the bile ducts.

Patients with elevated levels of tumor markers were excluded from the study (Table III).

\section{Discussion}

Retrograde cholangiopancreatography is a very important method for the diagnosis and treatment of pancreas, liver and biliary tract diseases including choledocholithiasis. It is, however, associated with a high risk of complications. The decision to perform ERCP should be preceded by a thorough analysis of the case, taking into account the patient's clinical condition, results of laboratory tests and imaging studies $[10,11,17]$.

Patients' clinical condition is the first and the most important parameter that indicates the disease. In the case of choledocholithiasis, the main clinical symptoms are: abdominal pain, yellowing of skin and sclerae, dark urine, discolored stools, and itching of the skin. These symptoms are not pathognomonic for choledocholithiasis. They may be associated with other diseases of biliary tract, liver or pancreas. Therefore, the diagnosis of choledocholithiasis cannot be based only on clinical signs and symptoms. In our study, the accuracy of diagnosis made on the basis of clinical symptoms only, without accompanying abnormalities in imaging or laboratory tests, was 50\%.
The chances for accurate diagnosis of choledocholithiasis greatly increase if clinical symptoms occur together with laboratory abnormalities typical for cholestasis. In the present study, in patients with both clinical symptoms and increased levels of transaminases, GGT, ALP and conjugated bilirubin, ERCP confirmed the presence of choledocholithiasis in $80 \%$ of the cases, despite the lack of pathology in imaging studies.

Our study proved that if the suspicion of choledocholithiasis is based on clinical symptoms, accompanied by both laboratory and imaging abnormalities, the ERCP procedure confirms the occurrence of choledocholithiasis in $100 \%$ of cases. In the study of Singla et al. [18] the diagnostic sensitivity of ERCP was estimated to be $92 \%$, with $100 \%$ specificity and positive predictive value.

Similar classification into three risk groups of choledocholithiasis (high, intermediate and low) based on the combination of clinical symptoms, laboratory findings and imaging results was applied by Williams et al. [10, 11, 16]. Our study confirmed their observation that in the high-risk group ERCP should be performed as a diagnostic and therapeutic procedure with concurrent removal of stones from the bile ducts. In our study in the intermediate-risk group, the presence of stones in the bile ducts was confirmed in $87 \%$. This result allows the patient to be qualified for ERCP. The authors of the aforementioned study confirmed choledocholithiasis in about $50 \%$ of patients from the intermediate risk group. They suggested that in this group of patients choledocholithiasis should be confirmed by other non-invasive tests (MRI). Patients from the low risk group, in which the diagnosis is based on clinical symptoms only with neither laboratory nor imaging confirmation, should not be exposed to potential complications resulting from diagnostic ERCP. The main reason why the ERCP procedure should be performed solely in patients with a high probability of choledocholithiasis (high and intermediate risk groups) is a high incidence of serious, often life-threatening complications. Cotton and Shimizu reported that 
the incidence of pancreatitis associated with diagnostic ERCP (i.e. without sphincterotomy) is 3-6\% $[19,20]$. According to Freeman et al., sphincterotomy increases the complication rate to $9.8 \%$. Despite the fact that interventional procedures such as ERCP reduce mortality and morbidity rates, it has been hypothesized that these procedures may also cause bile contamination and thus increase the incidence of cholangitis [21, 22].

In conclusion, ERCP is a sensitive method of choledocholithiasis diagnosis. This procedure combines upper gastrointestinal (GI) endoscopy with $\mathrm{X}$-rays, and its sensitivity is comparable to other imaging studies. The ERCP is superior to other diagnostic tests (US, EUS, CT, MRI) because of the possibility of performing a concurrent therapeutic procedure including the removal of stones from the bile ducts. However, one should consider the risk of severe, even life-threatening ERCP complications. Thus, qualification for this procedure should be preceded by a thorough analysis of every case. The qualification should be based on three groups of indications: patient's clinical condition, results of laboratory tests and imaging studies. The combination of clinical symptoms, laboratory results and imaging findings allows patients to be divided into three risk groups: high (positive clinical symptoms, positive results of laboratory and imaging tests), intermediate (positive clinical symptoms, positive laboratory results, negative results of imaging tests) and low (positive clinical symptoms, negative laboratory and imaging tests). Considering the risk of choledocholithiasis and patient's safety, ERCP should be performed in patients classified in the high or intermediate risk group.

\section{Acknowledgments}

The work was done in Gastrointestinal Endoscopy Laboratory, WAM University Hospital, Medical University of Lodz, Poland

\section{Conflict of interest}

The authors declare no conflict of interest.

\section{References}

1. Johnson AG, Hosking SW. Appraisal of the management of bile duct Stones. Br J Surg 1987; 74: 555-60.

2. Halldestam I, Enell EL, Kullman E, Borch K. Development of symptoms and complications in individuals with asymptomatic gallstones. Br J Surg 2004; 91: 734-8.

3. Kawai K, Akasaka Y, Murakami K, Tada M, Koli Y. Endoscopic sphincterotomy of the ampulla of Vater. Gastrointest Endosc 1974; 20: 148-51.

4. Classen M, Demling L. Endoscopic sphincterotomy of the papilla of Vater and extraction of stones from the choledochal duct. Dtsch Med Wochenschr 1974; 99: 496-7.
5. Fletcher DR. Changes in the practice of biliary surgery and ERCP during the introduction of laparoscopic cholecystectomy to Australia: their possible significance. Aust N Z J Surg 1994; 64: 75-80.

6. Livingston EH, Rege RV. Technical complications are rising as common duct exploration is becoming rare. J Am Coll Surg 2005; 201: 426-33.

7. Canto MI, Chak A, Stellato T, Sivak MV. Endoscopic ultrasonography versus cholangiography for the diagnosis of choledocholithiasis. Gastrointest Endosc 1998; 47: 439-48.

8. Norton SA, Alderson D. Prospective comparison of endoscopic ultrasonography and endoscopic retrograde cholangiopancreatography in the detection of bile duet stones. Br J Surg 1997; 84: 1366-9.

9. Carr-Locke DL. Therapeutic role of ERCP in the management of suspected common bile duct stones. Gastrointest Endosc 2002; 56: 170-4.

10. Williams EJ, Green J, Beckingham I, Parks R, Martin D, Lombard M. Guidelines on the management of common bile duct stones. Gut 2008; 57: 1004-21.

11. Frossard JL, Morel PM. Detection and management of bile duct stones. Gastrointest Endosc 210; 72: 808-16.

12. Aliperti G. Complications related to diagnostic and therapeutic endoscopic retrograde cholangiopancreatography. Gastrointest Endosc Clin N Am 1996; 6: 379-407.

13. Baillie J. Complications of endoscopy. Endoscopy 1994; 26: 185-203.

14. Freman ML, Nelson DB, Sherman S, et al. Complications of endoscopic biliary sphincterotomy. N Engl I Med 1996; 335: 909-18.

15. Arguedas MR, Dupont AW, Wilcox CM. Where do ERCP, endoscopic ultrasound, magnetic resonance cholangiopancreatography, and intraoperative cholangiography fit in the management of acute biliary pancreatitis? A decision analysis model. Am J Gastroenterol 2001; 96: 2892-9.

16. Choudari CO, Fogel E, Kalayci C, et al. Therapeutic biliary endoscopy. Endoscopy 1999; 31: 80-7.

17. Maple JT, Ben-Menachem T, Anderson MA, et al. The role of endoscopy in the evaluation of suspected choledocholithiasis. Gastrointest Endosc 2010; 71: 1-9.

18. Singla S, Gulrez A, Pawar G. Comparative study of diagnostic efficacy of ultrasound and ERCP in extrahepatic cholestatic jaundice. IJSIT 2014; 3: 041-047.

19. Cotton PB. ERCP. Gut 1977; 18: 316-41.

20. Shimizu S, Tada M, Kawai K. Diagnostic ERCP. Endoscopy 1994; 26: 88-92.

21. Attasaranya S, Fogel EL, Lehman GA. Choledocholithiasis, ascending cholangitis, and gallstone pancreatitis. Med Clin North Am 2008; 92: 925-60.

22. Yusoff IF, Barkun IS, Barkun AN. Diagnosis and management of cholecystitis and cholangitis. Gastroenterol Clin North Am 2003; 32: 1145-68. 